\title{
Multi-agent Quality of Bee Products Traceability Model Based on Roles
}

\author{
Yue $\mathrm{E}^{1}$, YePing Zhu ${ }^{1}$, and YongSheng $\mathrm{Cao}^{2}$ \\ ${ }^{1}$ Research Institute of Agriculture Information, Chinese Academy of Agricultural Science, \\ Key Laboratory of digital agricultural early warning technology of ministry of agricultural, \\ 12, ZhongGuanCunNanDaJie,Beijing, China \\ eyue@mail.caas.net. cn \\ ${ }^{2}$ Institute of Crop Science, Chinese Academy of Agricultural Science, \\ 12, ZhongGuanCunNanDaJie, Beijing, China
}

\begin{abstract}
Role is an important concept in research on how to develop method of Agent-Oriented software. In this paper, To study the background is bee product quality traceability system based on Multi-Agent, base on bee product quality critical control point, and describes a role-based multi-level framework of the method. Through Agent get different roles, and it get a group of services related to the process of quality of bee products tracing. At the same time, through the dynamic role change to realize the collaborative process between Agent management. Multi-Agent system model based on roles provide new methods and ideas about the control of the quality and safety of bee products.
\end{abstract}

Keywords: Role, MAS, Bee Produces, Collaboration, Traceability.

\section{Introduction}

Quality of bee products traceability including quality of tracking, quality tracing, the core of the information collection, tracking, monitoring and control. Bee Products (such as honey, Royal Jelly, etc.) from field to home, it have a number of links includes the acquisition of bee products, bee product processing, transport and sale, This process can be divided into bee breeding, daily management, honey collection, processing equipment, processing personnel, processing procedures, packaging, storage, transportation, honey varieties, environmental pollution, pest control treatment, collection tools, acquisition time, equipment, sanitation, materials, health status, technical levels and so on. Each link exists between each other the exchange of information. Due to geographical differences (the management are widely distributed in different regions), the time difference (there is a huge time difference about access to products, access to relevant information), making information management has become very complicated.

From an engineering perspective, the bee product quality tracking and traceability system is a wide-area Internet-based technology and distributed artificial intelligence system. Agent technology can be used to solve the distributed system, it has many good features based on Agent and Multi-Agent technology application system, such as 
initiative, intelligence, interaction, collaboration and mobility and so on. Therefore, the quality of bee products tracking and traceability system can be decomposed as a dynamic system, no matter how large the scale, in theory, the system can be broken down into a number of interrelated sub-systems (such as acquisition system, processing systems, transport systems, marketing systems, etc.). There are also relatively independent subsystems in quality management in the actual work, the bee for the production, acquisition, processing, transportation and sales departments have the corresponding entities.

On the other hand, As a result of its own characteristics, Critical Control Point(such as pests and diseases, medication, heavy metals) play a very important role in the quality of bee products tracking and traceability system, the advantages and disadvantages of these entities directly related to the quality of bee products. These entities can completed their own tasks and goals to achieve optimal, they have independent thinking and ability to fulfill its mandate, they can cognitive state that all other entities of the current, and can accept the request and orders of other entities to change their behavior mechanism.

In this paper, a multi-agent cooperative module is proposed, that is a role-based dynamic multi-agent cooperative module. This module introduces a role concept into the multi-agent cooperative system. It rebuilds a library of role information in every agent, thus realizing multi-agent dynamic cooperation.

\section{Role and Binding Mechanism}

In general, Agent for the operation of a common goal, each Agent must continually analyze their environment, and to interact with each Agent in a multi-agent systems. At any time, there is a number of different external stimulate and behaviors need it to decision-making, and require a lot of time and communication resources. No doubt that this complex multi-Agent Learning System. In this case, a good solution is to give each Agent to determine their respective roles, each Agent has its own characteristics, and collaboration with the Agent is dynamically generated, dynamic constraints, thereby reducing traffic, improving the efficiency of learning. Based on this thinking, the article proposes a role-based dynamic multi-agent collaboration system.

\subsection{Role}

Role concept from sociology, the basic point is limited to the role of individual behavior, the relationship between role limited the interaction between individuals in the system. In the study of multi-Agent systems, the role as a bridge connection with the micro-macro model, some researchers introduced the concept of the role of system analysis and design. In the multi-Agent system, to the role defined as follows ${ }^{[1]}$ :

1. From the conceptual point of view, the role is a constraint. In this restriction, Agent involved in some of the interactions and evolution in some way.

2. From the implementation point of view, roles are some of the properties and behavior and binding on the Agent. 
The current study suggests that the relationship between the role and Agent with the following typical properties:

1. Dynamic: In the Agent life cycle, we can add new role and undo the original role.

2. Dependence: role of the Agent can not exist independently, it must rely on an Agent.

3. Multiplicity: the same Agent may assume different roles in the same period, and the same Agent may also take different roles in different times.

As the Agent may assume different roles and bring a different point of view, the Agent reflect more comprehensive and detailed knowledge.

\subsection{Binding Mechanism}

To coordinate the implementation of multiple Agent in the collaborative environment is a collaborative mission of the basic problems. In the Agent team, each Agent responsible for one or more role, through the dynamic transformation of role, the team can more effective implementation, in order to adapt to the environmental changes, to improve overall system performance. Agent role binding allows multiple Agent cooperative coordination in the implementation of tasks. In the multi-Agent system, it can take dynamic binding mechanism to achieve the transformation of role. Role binding is divided into three types:

1. Role Assignment: Agent play a new role after it complete the current mission.

2. The role of redistribution: Agent interrupt the current role and play another role.

3. Role of the exchange: Two Agent exchange of role in the implementation.

\section{MAS Model Based on Role}

\subsection{Agent Model}

The model is multi-Agent collaborative based on the role. Functions of each part of Model are described below:

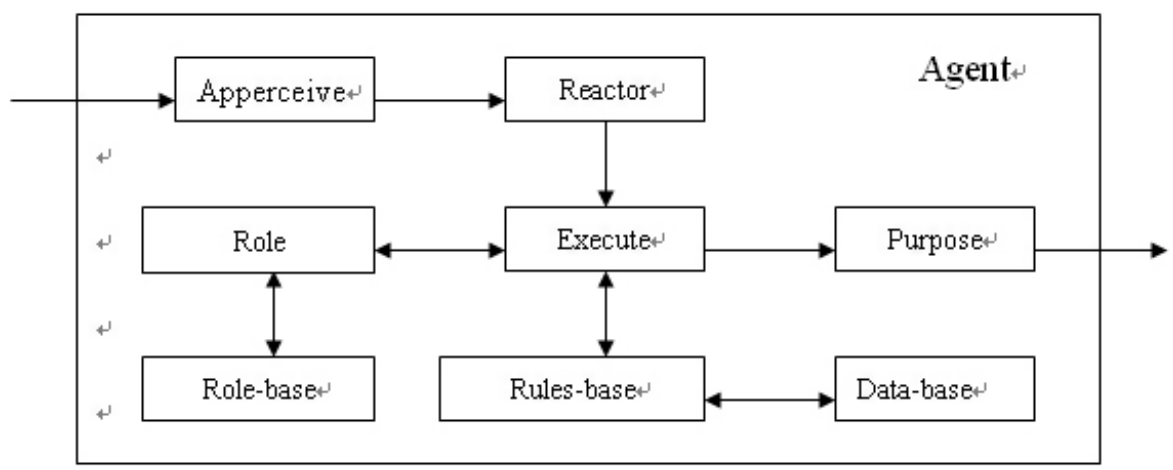

Fig. 1. Bee product quality traceability system Agent Model 
1. Apperceive is perceived external environment and internal state changes about Agent, and those changing circumstances the reactors.

2. Reactor receive the message from the apperceive, and those message submitted to the Execute.

3. Execute get message of the reactor, it obtained rules from Rules of conduct group, It obtained role from the role library if it need to add the role, It obtained data from the database if it need the data of Agent, and finally the results give Purpose.

4. Purpose is responsible for the task flow output.

5. Rules of conduct base is stored within the definition of the rules of the Agent.

6. Database stored data carried by Agent.

7. Role assignment is qualified for the role of verification and extraction and distribution about Agent.

8. The role base used to store the role definition.

Each activity was defined as a single Agent in the process of traceability of the quality of bee products, the Agent has a role, and to collaborate and interact with other Agent. For example, buyer can be defined as the buyer Agent, the Agent role is to receive beekeepers request and respond, if agreed to acquire, the buyer Agent continue to process the request to processed Agent, and then processed Agent with other relevant Agent interaction.

These Agent information exchange with the other Agent (such as beekeepers Agent, Sales Agent, etc.) through for communication interface, and then call the role-base specific role, analyse and process the exchange of information, thereby on exchange with the other Agent to respond. The exchange of information means that Agent of different task holders to the successful and satisfactory completion of an activity and exchange of information in the process of traceability of the quality of bee products. Behavior results is that the Agent based the role of the task, through the Agent exchange coordination with the requesting Agent, jointly with the other Agent to complete the activity behavior.

The Agent through the role-base to describe it play the role, an Agent may have one or more roles, so the Model can adapt to changing roles in traceability of the quality of bee products system, strengthen the model agility and expansibility. For example, Processing Agent plays the role of the original honey production tasks, if the business process changes, the processing Agent in addition to played the original honey production tasks, coupled with the acquisition of the role of the buy honey, then just in the process the role-base Agent added to the original honey acquisition role, do not need to modify the traceability model.

There are many Agent in traceability of the quality of bee products system, but in order to facilitate the discussion, we put them into an abstract class for the 3 main: decision-making Agent, Management Agent, Operation Agent.

This classification is based on the multi-level framework principle, and consider the consistency of role assignment. Multi-level framework features reflect the structure between Agents, Superior Agent control subordinate Agent behavior, multi-level framework of multi-Agent system can satisfy the real-time communication. 


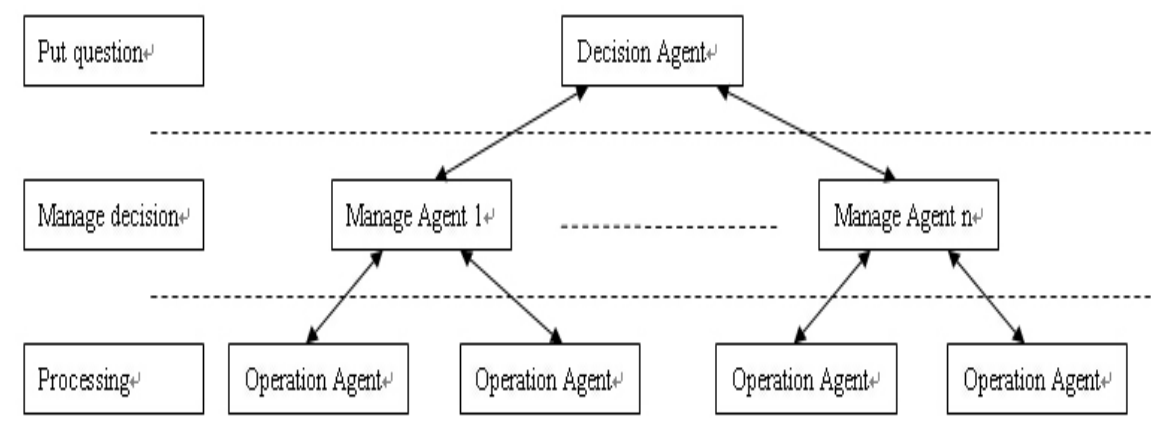

Fig. 2. Bee product quality traceability system architecture based on multi-level Framework

\subsection{The Definition of the Role in Agent}

Agent of social behavior is essential for building multi-Agent Collaboration model, the role of Agent behavior abstraction, reflected status and interaction in group (organization or society). So we must set up an appropriate role model to study the social of Agent.

In order to more fully and accurately reflect the role of the concepts, this paper defines the role model include obligation, authority, rule, activity, information, description.

1. Obligation is the role must fulfill obligations, it is the role in Agent assume tasks.

2. Authority is the role of responsibility for ensuring the realization, including the use of resources, access control permissions.

3. Rule is role must comply some norms, it also illustrates the role of supplementary obligations.

4. Activity is a group of private action about role, the implementation of these actions contribute to the role of its responsibilities. The so-called private action is implement these activities do not to involve interaction with other role.

5. Information is essential for each role domain knowledge and to help it achieve its role and function.

6. Description is the role of a conceptual description and introduction.

\subsection{Role Dynamic Transformation}

The beginning of the process of traceability of the quality of bee products, systems based on the demand assigned role to Agent. Implementation process, Agent interact with others agent through its role of responsibility and authority, provide the appropriate services. But as the environment or demand changes, it was asked to provide more services, but also need some new services, Therefore, the role needs to be transformation.

In order to achieve Agent dynamic transformation role in collaborative activities, the paper proposes role assignment methods to dynamic transformation role. One the one 
hand, the role assignment methods confirm role eligibility verification and appointed about Agent, On the other hand, the realization of the role to create, modify, delete, and matching management. The current role of the Agent set for the Role-Old, new role of dynamic transformation role for the Role-New, it called the goal role, and RoleData is role-base.

Based on the above description, describe the Agent role transformation process is as follows:

1. Agent request a group of services to the role assignment.

2. The role assignment search the role-base, and get a group similar role, and then recommend to the Agent.

3. The Agent select the most suitable role as a target role, and request play the role to the role assignment.

4. The role assignment validate conversion relationships and play the role of eligibility verification about the Agent.

5. The role assignment delete the original role assigned, and to establish new the role of the designated subject and target.

Thus, The role assignment help the Agent achieve the new target role and Agent dynamic transformation role in collaborative activities.

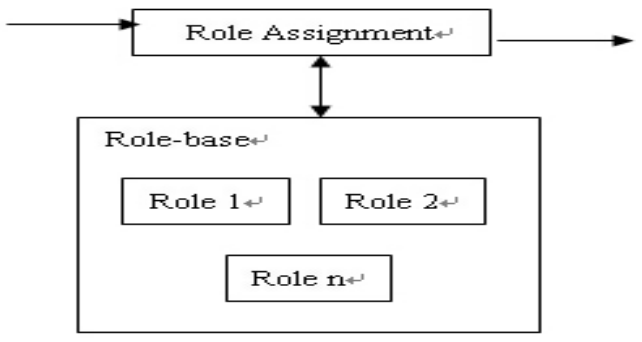

Fig. 3. Role Transformation

\section{Example}

* The first step:

The problem of input, Agent initialization. The initial state, the system environment including user Agent, Management Agent.

1. user interaction Agent is the system and user interface, receiving user input of information, when the task is completed, the results displayed to the user. It create the role list base on the user information, and dissemination through the role bulletin board, and then wait for other Agent apply for the role. Now we assume the role list base included drug residues, processing technology, disease, three role notice. 
2. Management Agent is a shared communication area, at the same time, it responsible for the management and provision of resources.

* The second step:

The resolve of decision making.

It has been in the environment of the Agent, inquiry into the environment role bulletin board, looking for own functions and resources to meet the requirements of the role and to determine the application for it own role. This time, Agent have a real function ( Role-based Agent), such as drug Agent, Processing Agent, Disease Agent.

* The third step:

The problem solving though Agent of collaboration.

1. The system started when all roles in role bulletin board application by Agent.

2. Due to the role of its "limitations", Agent not complete with its own functional role, such as Disease Agent running process, it need to generate better understanding of bee disease, its culture environmental information, but it is not ability to resolve, so the disease Agent apply to role bulletin board, on the one hand, it can join the existing operational environment to work together by generating new Agent (environmental Agent).On the other hand, completed Work Agent (such as: At this point, processing Agent has completed its mission in sleep state), according to "their" character (willingness) and the role-base functions, re-apply for new roles (environmental Agent) for system operation.

3. Management Agent real-time detection information in the role bulletin board in order to make the appropriate action (such as many Agent apply for a role, Agent work has been completed, etc.).

4. The results of each Agent through Management Agent to user interaction Agent.

* The fourth Step:

The results of information integration.User interaction Agent get results to the user form a friendly man-machine interface.

This cycle until the completion of the task.

With process management, supply chain management, value chain development and modern information and communication technology revolutionary. Traceability has been given more meaning. Traceability has evolved into a dynamic supply chain management and enterprise application integration based on the product track and trace, it become main tools about data management, process management, process control, decision support. Application of Agent technology, Users will need to find the information to inform Agent, and let it into the appropriate environment and collaboration and completion of treatment. Such as quality of bee products traceability system, it achieve an intelligent and automated so that consumers from tedious search for the goods, browse, compare, select and negotiate such links. Therefore, it has important research value that Multi-Agent Quality of Bee Products Traceability Model Based on Roles.

\section{Conclusion}

In this paper, multi-Agent system as a social organization, the tasks and objectives within the system is resolve the role, the Agent binding role to complete the mission 
and objectives. we achieve role transformation and collaboration based on role in this study, for further study of the relationship about Agent and role, roles and role relations and implementation mechanism.

\section{Acknowledgements}

This research was supported by National Scientific and Technical Supporting Programs Funded by Ministry of Science and Technology of China (nyhyzx07-041). National Natural Science Foundation about Agent-based quality control of agricultural products (60972154).

\section{References}

1. Li, Y., Ming, Z.W.: Intelligent and Cooperative Information Systems. Publishing of Electronics Industry, Beijing (2008)

2. Luger, G.F.: Artificial intelligence. Publishing of Electronics Industry, Beijing (2008)

3. Long, W., Yi, Z.: Key Techniques to Realize Cooperation of Mobile Agent in MAS Environment. School of Computer Science and Engineering 32(2), 158-163 (2009)

4. Trappey, A.J.C., Trappey, C.V., Hou, J.-l.: Mobile agent technology and application for online global logistic services. Industrial Management \& Data System 104(1/2), 169-183 (2008)

5. Ming, Z.J., Peng, Z.X.: An agent-oriented requirement analysis and modeling method 4, 33-35 (November 2009)

6. Jing, Z.: Bee product quality and safety analysis of the key technology research and development. China Apiculture 57(12), 30-32 (2006)

7. DeLoach, S.A.: Engineering Organization-based Multiagent Systems. In: Garcia, A., Choren, R., Lucena, C., Giorgini, P., Holvoet, T., Romanovsky, A. (eds.) SELMAS 2005. LNCS, vol. 3914, pp. 109-125. Springer, Heidelberg (2006)

8. Chang, M.-H., Harrington Jr., J.E.: Agent-based models of organizations. In: Handbook of Computational Economics II (2006)

9. Yi, S.C.: Computing based on Agent. Publishing of Tsinghua University (2007)

10. Zhi, S.Z.: Advanced Artificial Intelligence. Publishing of Science (2006) 